\title{
DETERMINACIÓN DE LA ELASTICIDAD DE LA DEMANDA ALIMENTARIA EN ECUADOR EN EL PERIODO 2013
}

\author{
DETERMINATION OF THE ELASTICITY OF FOOD DEMAND \\ IN ECUADOR FOR THE PERIOD 2013
}

\section{CARLOS HENRÍQUUZ HENRÍQUEZ ${ }^{1}$, CÉSAR FREIRE QUINTERO2 ${ }^{2}$ JUDITH MORAN PEÑA ${ }^{3}$}

\begin{tabular}{ll}
\hline 1 & Universidad Católica de Santiago de Guayaquil \\
carlos.henriquez@cu.ucsg.edu.ec \\
2. & Universidad Católica de Santiago de Guayaquil \\
3 & Estudiante de la Carrera de Economía, Universidad Católica de Santiago de Guayaquil
\end{tabular}

RESUMEN

El objetivo de la investigación es determinar la elasticidad de la demanda de productos alimenticios como el arroz blanco, la cebolla, el aceite vegetal, la sal y azúcar, productos de consumo frecuente en la mayoría de hogares. Dicho análisis se lo realizó mediante la aplicación de regresiones logarítmicas para poder determinar un coeficiente que detalle la variación porcentual de consumo en función de la variación porcentual del precio, la misma que permitió evidenciar un comportamiento inelástico, es decir, a medida que aumenta porcentualmente el precio, el consumo se reduce en menor cuantía porcentual.

PALABRAS CLAVE: ingresos, remuneración, elasticidad, precio de la demanda, modelo lineal, logaritmos.
ABSTRACT

The aim of the research is to determine the elasticity of demand for food products such as white rice, onion, vegetable oil, salt and sugar, which are found in most homes. This analysis would take place by applying logarithmic regression to determine a coefficient that details the percentage change in consumption depending on the percentage change in price, and this technique allowed to demonstrate an inelastic behavior, as a percentage price increases consumption is reduced to a lesser percentage rate.

KEYWORDS: income, compensation, price elasticity of demand, linear model, logarithms. 
INTRODUCCIÓN

Constantemente se busca implementar medidas de control de precio en el mercado de alimentos, y al ser este muy sensible al poder adquisitivo de las personas se deben adoptar políticas técnicas que permitan minimizar el impacto social. La sociedad puede afectarse en gran magnitud ante variaciones del precio de los alimentos, principalmente de aquellos que son más representativos en su inventario de despensa.

Actualmente, las políticas de control de precio sobre los alimentos van enfocadas a tratar de buscar un equilibrio entre vendedores y consumidores, de tal manera que se garantice la rentabilidad del oferente y no se distorsione la capacidad de consumo de la población.

El mercado de alimentos es poco sensible ante variaciones deprecios dado que su consumoes vital e irremplazable para la sociedad en su conjunto. Debido a esa importancia se pretende determinar la elasticidad de la demanda de alimentos en el Ecuador mediante el uso de técnicas estimativas que permitan generar una herramienta eficaz para el diseño de políticas públicas.

ANTECEDENTES

La teoría económica del comportamiento del consumidor se ha ampliado también mediante el desarrollo de sistemas completos de ecuaciones de demanda, que comprenden la asignación del presupuesto del consumidor a un grupo de categorías o productos o la asignación del gasto total en alimentación a varios productos genéricos. Esta especificación supone un análisis del comportamiento del consumidor más coherente que el análisis aislado de la demanda de productos específicos (Stiglitz, 2004).

Un aspecto relevante y útil para la realización de las estimaciones es que el uso de encuestas de hogares como fuente de datos podría generar una restricción en la realidad de los hogares al momento de realizar los cálculos, ya que algunos hogares no pueden consumir ciertos grupos de alimentos por lo que resultaría en un valor cero para la variable dependiente (Berges, Miriam \& Casellas, 2002).

Sin embargo, un desarrollo más reciente ha demostrado, mediante simulación bajo el Método de Montecarlo, que en el procedimiento en dos etapas que se propone para las ecuaciones sistemas con variables dependientes limitadas, los rendimientos de estimaciones son consistentes y se comportan mejor que otros propuestos con la inclusión de más variables explicativas (Cox \& Wohlgenant, 2006).

En cuanto a la calidad en el ajuste de precios, el mismo fue considerado para estimar funciones de demanda de alimentos de Argentina. Se comenta la importancia de ajustar los precios de las diferencias en cuanto a percepciones entre los hogares, que inclusive podrían registrar una variación en la percepción de precios. Este tipo de análisis debe ser implementado siguiendo este enfoque, en los ajustes de precios se realiza una regresión de los precios imputados sobre las características sociales y demográficas seleccionadas (Fabiosa \& Jensen, 2003).

Es necesario recalcar que el ajuste de precios se ha utilizado para Argentina en la realización de las estimaciones siguiendo el enfoque presentado en Paraguay y Bolivia, donde no hubo características sociales y demográficas de los hogares disponibles en la base de datos, razón por la cual el ajuste de calidad en ajustes de precios no era posible, lo que conllevó a la utilización de los precios implícitos, y para los casos de no compra, se utilizó la media ponderada de los precios regionales como el precio correspondiente (García, 2006).

$\mathrm{Al}$ analizar los modelos de demanda se requiere considerar aspectos restrictivos acerca de la conducta del consumidor para construir un sistema completo y adecuado de demanda, ya que de otra manera se debería tener información completa de precios y cantidades de todos los bienes (Heckman, 2004).

En cuanto al uso de la información que reposa en las encuestas nacionales se debe tener en consideración que hay que obviar el interés investigativo en centrarse solamente en un grupo pequeño de bienes y más bien, considerar la demanda de otros productos, observando disponibilidad de información; si es que no existiera, se debe hacer uso de sistemas incompletos de demanda (Hein \& Wessells, 2010).

Un sistema incompleto de demanda puede solucionar el problema de información en cuanto a precios y cantidades de los productos consumidos y además puede contribuir a la búsqueda de un modelo que incluya variables extremadamente necesarias (Lanfranco, 2005).

La derivación de ecuaciones de demanda a partir de solo alguno de los bienes consumidos, puede efectuarse de dos maneras. Una de ellas es asumir la separabilidad de la función de utilidad y derivar ecuaciones de demanda condicionadas. La otra opción es especificar directamente un sistema de demanda y luego 
tratar de reconciliarlo con la teoría económica (Lanfranco, 2004).

La maximización de una función de utilidad sujeta a una restricción presupuestaria da origen a un sistema completo de funciones de demanda con ciertas propiedades. Si un subconjunto de este conjunto total de funciones de demanda es separado del resto, provocaría un sistema incompleto de demanda y por ende las propiedades cambiarían de manera ligera (Shonkwiler \& Yen, 2009).

Es necesario conocer si existe un listado exhaustivo de propiedades sobre un conjunto de funciones de demanda que garantice la recobrabilidad de las preferencias del consumidor, si esto sucede se puede asumir que existe una función de utilidad a partir del cual se derivan otras funciones (Lanfranco, 2004).

En cuanto a la elasticidad precio se puede concluir que refleja la variación porcentual en la cantidad consumida del bien dado una variación de $1 \%$ en el precio del mismo bien, así mismo la elasticidad cruzada mide la variación en el consumo de un bien ante la variación de $1 \%$ en el precio de otro bien (Ortiz, 2002).

En el uso de la base de datos se puede esperar que los datos de corte transversal tengan una parte representativa de valores cero debido a la no frecuencia de compra, la preferencia de los consumidores o que simplemente no se adquieran los bienes al precio o al nivel de ingreso obtenido, por lo que se le debe dar el trato estadístico adecuado (Fabiosa \& Jensen, 2003). Para este tipo de modelos se debe considerar el uso de técnicas a través de modelos logarítmicos donde se obtiene una variable independiente y una dependiente para obtener una relación entre la variación porcentual de una, con la variación porcentual de la otra. (García, 2006)

El objetivo de la investigación es determinar la elasticidad de los productos alimenticios que son más representativos en el inventario de despensa de los hogares del Ecuador.

\section{MATERIALES Y MÉTODOS}

La investigación realizada fue de tipo concluyente sobre la base del uso de fuentes secundarias, para lo cual se procedió a realizar un análisis descriptivo y de tipo econométrico, a través de la aplicación de logaritmos con el objetivo de determinar la elasticidad y verificar cual es la relación existente entre la variación porcentual de los precios y la variación del nivel de consumo en los alimentos.
Para el desarrollo del modelo econométrico se realizó una estimación de la elasticidad precio de la demanda, a través de la aplicación logarítmica a la relación funcional entre precio y demanda por cada uno de los bienes de consumo involucrados en el análisis de tal manera que:

$$
\mathrm{Q}(\mathrm{p})=\beta \_1-\beta \_2 \mathrm{p}
$$

Representa la función de demanda por lo que se procedió a la aplicación logarítmica y se obtiene:

$$
\ln (\mathrm{Q}(\mathrm{p}))=\beta \_1-\beta \_2 \ln (\mathrm{p})
$$

Siendo esta la función ajustada para efectos de la estimación de regresión.

$$
\Delta \ln (\mathrm{Q}(\mathrm{p}))=\beta \_2 \Delta \ln (\mathrm{p})
$$

Esta función representó el cambio porcentual del consumo ante un cambio porcentual del precio, representando la estimación de la elasticidad de la demanda.

\section{TABLA 1. VARIABLES A UTILIZARSE}

Nivel de educación del jefe de hogar

Sexo del jefe de hogar

Región geográfica

Cantidad de miembros del hogar

\section{Ingreso de Hogar}

Edad del Jefe de Hogar

Tamaño del hogar

Nivel de ingreso elevado al cuadrado

Nivel de ingreso multiplicado por tamaño del hogar

En cuanto al uso de materiales se emplearon bases de datos provenientes del INEC, Programa Statgraphics, Stata y SPSS. Se manejó datos secundarios proporcionados por el Instituto Nacional de Estadística y Censos, a través de la Encuesta Nacional de Ingresos y Gastos.

Aceite vegetal de palma africana

RESULTADOS

Regresión Simple - LOG (consaceite) vs. LOG (pestrelataceit)

Variable dependiente: LOG (consaceite)

Variable independiente: LOG (pestrelataceit)

Lineal: $\mathrm{Y}=\mathrm{a}+\mathrm{b}^{*} \mathrm{X}$ 
TABLA 2. COEFICIENTES

\begin{tabular}{lrrrr|} 
& $\begin{array}{r}\text { MÍ́NIMOS } \\
\text { CUADRADOS }\end{array}$ & ESTÁNDAR & ESTADÍSTICO & \\
\hline PARÁMETRO & ESTIMADO & ERROR & T & VALOR-P \\
\hline Intercepto & 0,345296 & 0,0135531 & 25,4772 & 0,0000 \\
\hline Pendiente & $-0,447633$ & 0,00444277 & $-100,755$ & 0,0000 \\
\hline
\end{tabular}

Fuente: ENIGHUR.

TABLA 3. ANÁLISIS DE VARIANZA

\begin{tabular}{lrrrrr} 
FUENTE & $\begin{array}{r}\text { SUMA DE } \\
\text { CUADRADOS }\end{array}$ & GL & $\begin{array}{r}\text { CUADRADO } \\
\text { MEDIO }\end{array}$ & RAZÓN-F & VALOR-P \\
\hline Modelo & 1864,52 & 1 & 1864,52 & 10151,65 & 0,0000 \\
\hline Residuo & 2404,93 & 13094 & 0,183666 & & \\
\hline Total (Corr.) & 4269,44 & 13095 & & & \\
\hline
\end{tabular}

Fuente: ENIGHUR.

La salida muestra los resultados de ajustar un modelo lineal para describir la relación entre LOG (consaceite) y LOG (pestrelataceit).

La ecuación del modelo ajustado es:

LOG $($ consaceite $)=$

$$
0,345296-0,447633^{*} \mathrm{LOG} \text { (pestrelataceit) }
$$

Puesto que el valor-P en la tabla ANOVA es menor que 0,05 , existe una relación estadísticamente significativa entre LOG (consaceite) y LOG (pestrelataceit) con un nivel de confianza del $95,0 \%$.

El estadístico R-Cuadrada indica que el modelo ajustado explica $43,6712 \%$ de la variabilidad en LOG (consaceite). El coeficiente de correlación es igual a -0,660842, indicando una relación moderadamente fuerte entre las variables. El error estándar del estimado indica que la desviación estándar de los residuos es 0,428563 .

ELASTICIDAD ARROZ BLANCO

Regresión Simple - LOG (consarroz) vs. LOG (pestimadrel)

Variable dependiente: LOG (consarroz)

Variable independiente: LOG (pestimadrel)

Lineal: $\mathrm{Y}=\mathrm{a}+\mathrm{b}^{*} \mathrm{X}$

TABLA 4. COEFICIENTES DE LOG (CONSARROZ) VS. LOG (PESTIMADREL)

\begin{tabular}{lrrrr} 
& \multicolumn{2}{l}{$\begin{array}{l}\text { MÍNIMOS } \\
\text { CUADRADOS }\end{array}$} & ESTÁNDAR & ESTADÍSTICO \\
\\
\hline PARÁMETRO & ESTIMADO & ERROR & T & VALOR-P \\
\hline Intercepto & 1,831 & 0,00949781 & 192,782 & 0,0000 \\
\hline Pendiente & $-0,513016$ & 0,00473234 & $-108,407$ & 0,0000 \\
\hline
\end{tabular}

Fuente: ENIGHUR.
TABLA 5. ANÁLISIS DE VARIANZA DE LOG (CONSARROZ) VS. LOG (PESTIMADREL)

\begin{tabular}{lrrrrr} 
FUENTE & $\begin{array}{r}\text { SUMA DE } \\
\text { CUADRADOS }\end{array}$ & GL & $\begin{array}{r}\text { CUADRADO } \\
\text { MEDIO }\end{array}$ & RAZÓN-F & VALOR-P \\
\hline Modelo & 3956,42 & 1 & 3956,42 & 11751,99 & 0,0000 \\
\hline Residuo & 3828,49 & 11372 & 0,336659 & & \\
\hline Total (Corr.) & 7784,91 & 11373 & & & \\
\hline
\end{tabular}

Fuente: ENIGHUR.

La salida muestra los resultados de ajustar un modelo lineal para describir la relación entre LOG (consarroz) y LOG (pestimadrel).

La ecuación del modelo ajustado es:

LOG $($ consarroz $)=$

$$
1,831-0,513016 * \text { LOG (pestimadrel) }
$$

Puesto que el valor-P en la tabla ANOVA es menor que 0,05 , existe una relación estadísticamente significativa entre LOG (consarroz) y LOG (pestimadrel) con un nivel de confianza del $95,0 \%$.

El estadístico R-Cuadrada indica que el modelo ajustado explica 50,8216\% de la variabilidad en LOG (consarroz). El coeficiente de correlación es igual a -0,712893, indicando una relación moderadamente fuerte entre las variables. El error estándar del estimado indica que la desviación estándar de los residuos es 0,580224. Este valor puede usarse para construir límites de predicción para nuevas observaciones.

ELASTICIDAD AZÚCAR BLANCA Regresión Simple - LOG (consazuc) vs. LOG (preazucrelat)

Variable dependiente: LOG (consazuc)

Variable independiente: LOG (preazucrelat) Lineal: $\mathrm{Y}=\mathrm{a}+\mathrm{b}^{*} \mathrm{X}$

TABLA 6 COEFICIENTE LOG (CONSAZUC) VS. LOG (PREAZUCRELAT)

\begin{tabular}{lrrrr} 
& $\begin{array}{r}\text { MÍNIMOS } \\
\text { CUADRADOS }\end{array}$ & ESTÁNDAR & ESTADístico & \\
\hline PARÁmetro & ESTIMADO & ERROR & T & VALOR-P \\
\hline Intercepto & 0,987042 & 0,00919385 & 107,359 & 0,0000 \\
\hline Pendiente & $-0,564198$ & 0,0048514 & $-116,296$ & 0,0000 \\
\hline Fuente: ENIGHUR. & & & & \\
\hline
\end{tabular}

TABLA 7. ANÁLISIS DE VARIANZA

\begin{tabular}{lrrrrr}
\multicolumn{1}{c}{ FUENTE } & $\begin{array}{r}\text { SUMA DE } \\
\text { CUADRADOS }\end{array}$ & GL & $\begin{array}{r}\text { CUADRADO } \\
\text { MEDI0 }\end{array}$ & RAZÓN-F & VALOR-P \\
\hline Modelo & 4301,4 & 1 & 4301,4 & 13524,74 & 0,0000 \\
Residuo & 4493,26 & 14128 & 0,318039 & & \\
\hline Total (Corr.) & 8794,65 & 14129 & & & \\
\hline
\end{tabular}

Fuente: ENIGHUR. 
La salida muestra los resultados de ajustar un modelo lineal para describir la relación entre LOG (consazuc) y LOG (preazucrelat).

La ecuación del modelo ajustado es:

LOG $($ consazuc $)=$

$$
\text { 0,987042-0,564198*LOG (preazucrelat) }
$$

Puesto que el valor-P en la tabla ANOVA es menor que 0,05 , existe una relación estadísticamente significativa entre LOG (consazuc) y LOG (preazucrelat) con un nivel de confianza del $95,0 \%$.

El estadístico R-Cuadrada indica que el modelo ajustado explica $48,9092 \%$ de la variabilidad en LOG (consazuc). El coeficiente de correlación es igual a -0,699351, indicando una relación moderadamente fuerte entre las variables. El error estándar del estimado indica que la desviación estándar de los residuos es 0,56395. Este valor puede usarse para construir límites de predicción para nuevas observaciones.

ELASTICIDAD CEBOLLA PAITEÑA COLORADA Regresión Simple - LOG consuceb) vs. LOG (prelatceb)

Variable dependiente: LOG (consuceb) Variable independiente: LOG (prelatceb) Lineal: $\mathrm{Y}=\mathrm{a}+\mathrm{b}^{*} \mathrm{X}$

\section{TABLA 8. COEFICIENTE LOG (CONSUCEB) VS LOG (PRELATCEB)}

\begin{tabular}{lrrrr} 
& $\begin{array}{r}\text { MÍNIMOS } \\
\text { CUADRADOS }\end{array}$ & ESTÁNDAR & EStAdístico & \\
\hline PARÁMETRO & ESTIMADO & ERROR & T & VALOR-P \\
\hline Intercepto & 0,551632 & 0,00811324 & 67,9915 & 0,0000 \\
\hline Pendiente & $-0,634663$ & 0,00495282 & $-128,142$ & 0,0000 \\
\hline
\end{tabular}

Fuente: ENIGHUR.

TABLA 9. ANÁLISIS VARIANZA LOG (CONSUCEB) VS LOG (PRELATCEB)

\begin{tabular}{lrrrrr|}
\hline FUENTE & \multicolumn{1}{c}{$\begin{array}{l}\text { SUMA DE } \\
\text { CUADRADOS }\end{array}$} & \multicolumn{1}{c}{ GL } & \multicolumn{1}{c}{$\begin{array}{l}\text { CUADRADO } \\
\text { MEDIO }\end{array}$} & RAZÓN-F & VALOR-P \\
\hline Modelo & 4952,53 & 1 & 4952,53 & 16420,32 & 0,0000 \\
\hline Residuo & 3443,48 & 11417 & 0,30161 & & \\
\hline $\begin{array}{l}\text { Total } \\
\text { (Corr.) }\end{array}$ & 8396,01 & 11418 & & & \\
\hline
\end{tabular}

Fuente: ENIGHUR.

La salida muestra los resultados de ajustar un modelo lineal para describir la relación entre LOG (consuceb) y LOG (prelatceb). La ecuación del modelo ajustado es
LOG $($ consuceb $)=0,551632-0,634663^{*}$ LOG (prelatceb)

Puesto que el valor-P en la tabla ANOVA es menor que 0,05 , existe una relación estadísticamente significativa entre LOG (consuceb) y LOG (prelatceb) con un nivel de confianza del 95,0\%.

El estadístico R-Cuadrada indica que el modelo ajustado explica 58,9867\% de la variabilidad en LOG (consuceb). El coeficiente de correlación es igual a -0,768028, indicando una relación moderadamente fuerte entre las variables. El error estándar del estimado indica que la desviación estándar de los residuos es 0,54919. Este valor puede usarse para construir límites de predicción para nuevas observaciones.

ELASTICIDAD FIDEO (LAZOS, CONCHAS, LETRA) Regresión Simple - LOG (consfideo) vs. LOG (prefideorel)

Variable dependiente: LOG (consfideo) Variable independiente: LOG (prefideorel) Lineal: $\mathrm{Y}=\mathrm{a}+\mathrm{b}^{*} \mathrm{X}$

TABLA 10. COEFICIENTE DE LOG (CONSFIDEO) VS LOG (PREFIDEOREL)

\begin{tabular}{lrrrr} 
& $\begin{array}{r}\text { MÍNIMOS } \\
\text { CUADRADOS }\end{array}$ & ESTÁNDAR & ESTADíSTICO & \\
\hline PARÁMEtRO & ESTIMADO & ERROR & T & VALOR-P \\
\hline Intercepto & 0,56317 & 0,0139259 & 40,4405 & 0,0000 \\
\hline Pendiente & $-0,64719$ & 0,00740501 & $-87,399$ & 0,0000 \\
\hline
\end{tabular}

Fuente: ENIGHUR.

TABLA 11. ANÁLISIS DE VARIANZA LOG (CONSFIDEO) VS LOG (PREFIDEOREL)

\begin{tabular}{lrrrrr|}
\hline FUENTE & $\begin{array}{r}\text { SUMA DE } \\
\text { CUADRADOS }\end{array}$ & GL & $\begin{array}{r}\text { CUADRADO } \\
\text { MEDIO }\end{array}$ & RAZÓN-F & VALOR-P \\
\hline Modelo & 2777,85 & 1 & 2777,85 & 7638,59 & 0,0000 \\
\hline Residuo & 2960,56 & 8141 & 0,36366 & & \\
\hline Total (Corr.) & 5738,4 & 8142 & & & \\
\hline
\end{tabular}

Fuente: ENIGHUR.

La salida muestra los resultados de ajustar un modelo lineal para describir la relación entre LOG (consfideo) y LOG (prefideorel).

La ecuación del modelo ajustado es:

LOG $($ consfideo $)=$

$$
0,56317-0,64719^{*} \text { LOG (prefideorel) }
$$

Puesto que el valor-P en la tabla ANOVA es menor que 0,05 , existe una relación estadísticamente significativa entre LOG (consfideo) y LOG (prefideorel) con un nivel de confianza del $95,0 \%$. 
El estadístico R-Cuadrada indica que el modelo ajustado explica 48,408\% de la variabilidad en LOG (consfideo). El coeficiente de correlación es igual a-0,695759, indicando una relación moderadamente fuerte entre las variables. El error estándar del estimado indica que la desviación estándar de los residuos es 0,603042. Este valor puede usarse para construir límites de predicción para nuevas observaciones, seleccionando la opción de Pronósticos del menú de texto.

ELASTICIDAD SAL REFINADA

Regresión Simple - LOG (conssal) vs. LOG (precsalrelat)

Variable dependiente: LOG (conssal)

Variable independiente: LOG (precsalrelat)

Lineal: $\mathrm{Y}=\mathrm{a}+\mathrm{b}^{*} \mathrm{X}$

TABLA 12 COEFICIENTES LOG (CONSSAL) VS LOG (PRECSALRELAT)

\begin{tabular}{lrrrr} 
& $\begin{array}{r}\text { MÍNIMOS } \\
\text { CUADRADOS }\end{array}$ & ESTÁNDAR & ESTADÍSTICO & \\
\hline PARÁMEtRO & ESTIMADO & ERROR & T & VALOR-P \\
\hline Intercepto & 0,56317 & 0,0139259 & 40,4405 & 0,0000 \\
\hline Pendiente & $-0,64719$ & 0,00740501 & $-87,399$ & 0,0000 \\
\hline
\end{tabular}

Fuente: ENIGHUR.

TABLA 13. ANÁLISIS VARIANZA

\begin{tabular}{lrrrrr}
\hline FUENTE & $\begin{array}{r}\text { SUMA DE } \\
\text { CUADRADOS }\end{array}$ & GL & $\begin{array}{r}\text { CUADRADO } \\
\text { MEDI0 }\end{array}$ & RAZÓN-F & VALOR-P \\
\hline Modelo & 640,703 & 1 & 640,703 & 2510,58 & 0,0000 \\
\hline Residuo & 1181,07 & 4628 & 0,255201 & & \\
\hline Total (Corr.) & 1821,77 & 4629 & & & \\
\hline
\end{tabular}

Fuente: ENIGHUR.

La salida muestra los resultados de ajustar un modelo lineal para describir la relación entre LOG (conssal) y LOG (precsalrelat).

La ecuación del modelo ajustado es:

LOG $($ conssal $)=$

$-0,523675-0,44387^{*}$ LOG (precsalrelat)

Puesto que el valor-Pen la tabla ANOVA es menor que 0,05 , existe una relación estadísticamente significativa entre LOG (conssal) y LOG (precsalrelat) con un nivel de confianza del 95,0\%.

El estadístico R-Cuadrada indica que el modelo ajustado explica $35,1692 \%$ de la variabilidad en LOG (conssal). El coeficiente de correlación es igual a -0,593036, indicando una relación moderadamente fuerte entre las variables. El error estándar del estimado indica que la desviación estándar de los residuos es 0,505174.

\section{DISCUSIÓN DE LOS RESULTADOS}

Los resultados obtenidos simbolizan una herramienta para respaldar las decisiones de política pública enfocadas al control de precio de alimentos, los datos hacen referencia a la elasticidad precio de la demanda calculada bajo una perspectiva técnica que mostró un comportamiento de inelasticidad.

Los resultados se relacionan en gran magnitud a los conocimientos existentes, dado que para cada uno de los bienes analizados se evidenció un comportamiento inelástico, lo que respalda la teoría que ciertos productos no tienen un nivel de sustitución perfecta, y esto sugiere adoptar medidas públicas que garanticen el poder adquisitivo de la sociedad.

El trabajo realizado respalda conocimientos ya adquiridos y así mismo proporciona conocimiento técnicos sobre la base de las herramientas econométricas que permitieron estimar el modelo, dado que actualmente se podrá conocer con exactitud las variaciones porcentuales del consumo ante una variación porcentual del precio de los bienes analizados.

Así mismo se sugiere el uso de nuevas metodologías que permitan establecer la dinámica el comportamiento de todo el mercado de alimentos a nivel nacional y regional para poder tener un comparativo de los niveles de sensibilidad en el mercado de alimentos.

En los resultados se evidencia la relación existente entre el nivel de precios y el consumo de los alimentos, principalmente aquellos que son más representativos a nivel de la canasta básica, y que se encontraron en un momento determinado y formaron parte del inventario de alimentos registrados por la Encuesta Nacional de Ingresos y Gastos de los Hogares Urbanos y Rurales (ENIGHUR).

Se demostró el comportamiento inverso que existe entre estas dos variables, de tal manera que al existir una variación porcentual en los niveles de precio se va a efectuar una reducción porcentual del nivel de consumo de alimentos. Por tal motivo es importante que las políticas dirigidas al control de precios de alimentos estén formuladas bajo una perspectiva técnica para garantizar la no restricción en el consumo de los hogares ante una decisión pública. 
CONCLUSIONES

Sobre la base del análisis realizado se puede concluir que el consumo de alimentos como el aceite, el arroz, la azúcar blanca, la cebolla, el fideo y la sal refinada son sensibles ante variaciones del precio y presentan un comportamiento inelástico, lo que simboliza que para estos producto es muy limitada la presencia de bienes sustitutos.

La sal refinada, el azúcar refinado, el aceite vegetal, los fideos, el arroz y la cebolla son productos que constan de manera común o repeti-

\section{REFERENCIAS BIBLIOGRÁFICAS}

Berges, Miriam and Casellas Karina (2002) "a demand system analysis of food for poor and non poor households. the case of Argentina" manuscript, Universidad Nacional de Mar del Plata, Argentina.

Cox, t and Wohlgenant, M (2006) "prices and quality effects in cross - sectional demand analysis". american journal of agricultural economics vol 68 nro 4. november 2006.

Fabiosa, j. and Helen H. Jensen (2003) "usefulness of incomplete demand model in censored demand system estimation" american agricultural economics asociation annual meeting, montreal, quebec, canada, july 2730, 2003.

García, Carlos I. (2006) “a comparative study of household demand for meats by U.S. Hispanics" Msc thesis Louisiana state university.

Heckman, J.J. (2004) "sample selection bias as a specification error.” econometrica47:153-162.

Hein, D and Wessells, C (2010) "demand system estimation with microdata: a censored regression approach" journal of business and economics statistics, july 2010. tiva en el $50 \%$ de los hogares de la muestra, por tal motivo se revela la importancia en la toma de decisiones públicas respecto a la estabilidad de los precios de dichos alimentos.

\section{AGRADECIMIENTOS}

Mis sinceros agradecimientos a la Universidad Católica Santiago de Guayaquil, por su aporte a la investigación docente, a mi equipo de trabajo por su constante empeño en el desarrollo de este proyecto.

Lanfranco, Bruno (2004) "aspectos teóricos y estimación empírica de sistemas de demanda por alimentos". XXXV reunión anual de la asociación Argentina de Economía Agraria, noviembre de 2004, Mar del Plata, Argentina.

Lanfranco, Bruno (2005) "characteristics of Hispanic households' demand for meats: a comparison with other ethnic groups utilizing an incomplete system of censored equations" $\mathrm{PhD}$ dissertation, the university of Georgia.

Ortiz, H. (2002) "economía popular, economía solidaria, fuerza para el desarrollo humano en el Perú”, pág. 1, mimeo.

Stiglitz, J. (2004). Microeconomía (2a. ed.). Barcelona: Ariel

Singh, Vishal p., Hansen Karsten t., y Gupta Sachin (2005), "modeling preferences for common attributes in multicategory brand choice," journal of marketing research, 42 (mayo), 195-209.

Song, i. y Chintagunta, p. (2007): "a discretecontinuous model for multicategory purchase behavior of households", journal of marketing research, p. 959-612. 